\title{
LA INVESTIGACIÓN RURAL DEL SIGLO XXI EN ESPAÑA
}

\author{
Concepción Foronda Robles \\ Departamento de Geografía Humana, Universidad de Sevilla \\ foronda@us.es
}

\section{RESUMEN}

Con motivo de la celebración del XVI Coloquio de Geografía Rural «Investigando en Rural», COLORURAL, celebrado en Sevilla, mayo 2012, se analizan los progresos científicos desarrollados a partir de las tesis doctorales relacionadas con Geografía Rural leídas en el siglo XXI y los proyectos nacionales I+D de los últimos años (2008-2011) en las universidades españolas.

A la habitual dispersión temática que encierra «lo rural» se suma la disparidad de perspectivas de estudios. La producción científica plantea nuevos cambios en este proceso de globalización en el que se inserta la investigación. Post-productivismo, desarrollo rural, prestación de servicios o percepción del paisaje son algunos de los temas de los que se incluyen en dichas investigaciones.

Palabras clave: Geografía rural, producción científica, tesis doctoral, proyecto I+D.

\section{ABSTRACT}

To mark the conclusion of XVI Rural Geography Colloquium «Researching in Rural», COLORURAL, celebrated in Seville on May 2012, it's analyzed scientific advances developed in the spanish universities from Rural Geography doctoral thesis in $21^{\text {st }}$ century and the $\mathrm{R}+\mathrm{D}$ national projects in recent years (2008-2011).

The usual dispersion of the subject matter related with the concept of «rural» is added to the high variety of studies perspectives. The scientific production consider new challenges in this globalization process, where the research is inserted. Post-productivism, rural development, service delivery or perception of the landscape are some of the issues that will be included in future research works.

Key words: Rural Geography, scientific production, doctoral thesis, project R+D. 


\section{INTRODUCCIÓN}

La investigación reciente en Geografía ha mostrado un creciente interés en las formas que se lleva a cabo la ruralidad. Ésta ha experimentado un proceso de transformación y modernización. Calidad, innovación, biodiversidad, paisaje, patrimonio... son elementos esenciales. El despliegue de la información y comunicación, y de los servicios educativos y sociales ha renovado el mundo rural y ha proporcionado una mayor diversificación económica y una mejora de la calidad de vida de sus habitantes. Los territorios rurales han dado en la última década un salto hacia la innovación tecnológica, también hacia la innovación social, con nuevas formas de relación entre los distintos actores del territorio.

Ante la necesidad de crear nuevas redes de transferencia, dentro del XVI Coloquio de Geografía Rural «Investigando en Rural» de la Asociación de Geógrafos Españoles celebrado en Sevilla, del 10 al 12 de mayo de 2012, el Comité Organizador decide dedicar una jornada en analizar la investigación rural del siglo XXI.

Por ello se presentan las tendencias actuales a través de los progresos científicos desarrollados a partir de las tesis doctorales leídas (2000-2011) y proyectos I+D de los últimos años (2008-2011) en las universidades españolas.

\section{LA PRODUCCIÓN CIENTIFICA A TRAVÉS DE LAS TESIS DOCTORALES}

La ciencia y la tecnología ejercen un gran papel en la historia del presente siglo, por su influencia directa en el desarrollo económico, político y cultural. Partiendo del supuesto que la tesis doctoral es un trabajo académico de iniciación científica, fuente de información que refleja el logro en su propio campo del saber, se considera de gran importancia la recopilación y su análisis. Las características metodológicas de las tesis doctorales, la orientación del director y la evaluación del tribunal garantizan el nivel de calidad de este tipo de trabajo de investigación, que constituye un buen indicador, no sólo cuantitativo sino cualitativo de la producción científica.

De acuerdo con la información procedente del Registro TESEO del Ministerio de Educación y Cultura disponible en http://www.educacion.gob.es/teseo, esta base de datos recoge las tesis doctorales defendidas en las universidades españolas, y permite la búsqueda por múltiples criterios: autor, director, título, resumen, universidad, departamento, curso académico y palabras clave (en base a tesauro).

Ya existen artículos que preceden a éste donde se recogen las tesis en Geografía leídas en los años noventa (Lázaro y Torres, 2002). Por ello, se han tomado las tesis de Geografía defendidas entre el año 2000 y 2011, registradas en TESEO, donde alguno de sus descriptores sea «geografía rural» (540402). En el período analizado se contabilizan 72 tesis.

En cuanto a la temática predominante se ha trabajado con el título de las mismas, sin juzgar la calidad de la investigación, ni entrar a fondo en ellas. Dicho análisis se ha llevado a cabo a través de un análisis cualitativo de datos textuales con el software Atlas.ti 5. Se procedió a complementar la interpretación de los datos de las redes obtenidas junto con la visualización de las nubes conceptuales generadas con todos los códigos resultantes, con ayuda del programa en línea Wordle. Con éste se busca resaltar de forma visual las coincidencias y frecuencias mayores de códigos en cada palabra. 


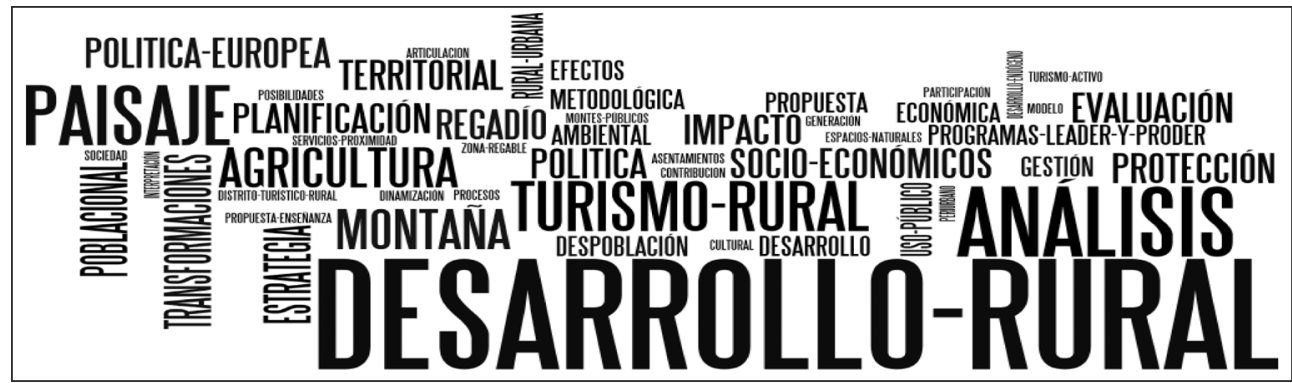

Fuente: Elaboración propia basada en Ministerio de Educación, Cultura y Deporte (2012).

Dicha representación queda agrupada en siete intervalos de tamaño atendiendo a la reiteración de las palabras que componen los títulos de las tesis rurales.

Aspectos históricos, teóricos, coyunturales, de interés reciente o presentes, han dado lugar a investigaciones de todo tipo. Muchos ruralistas españoles continúan trabajando sobre temas de interés tradicional, que son de actualidad. Otros, sin embargo, se hacen eco de los cambios ocurridos recientemente en el mundo rural español, de la aparición de nuevos tipos de agricultura, de las nuevas actividades y usos del suelo, de un espacio rural multifuncional donde ya no siempre la agricultura es la actividad principal (Age Rural, 2000).

Lo más llamativo es como la política europea de desarrollo rural, a través de los programas LEADER y PRODER iniciados en la década de los años 90, han dado significativos resultados académicos, a la vez del interés político-administrativo mostrado por el espacio rural. En este siglo XXI se ha convertido en una temática recurrente, asociada a los objetivos de la política comunitaria: revitalización de los territorios rurales a través de la participación de la población en las estrategias de desarrollo, diversificación económica o valorización de los recursos endógenos. De ahí que se hayan defendido tesis generalistas (Nieto Massot, 2007), para conseguir una mayor dinamización poblacional (Guru Puget, 2001), aceptando las potencialidades socioeconómicas (Astorga González, 2002; Mora Aliseda, 2006), y en especial en el turismo rural (Foronda Robles, 2002; Sparrier, 2005; García González, 2009; Solsona Monzonis, 2010). Otros planteamientos asociados son el papel jugado por el medio ambiente (Rubio Pastor, 2006), los servicios públicos (Escribano Pizarro, 2010), así como la construcción social en el desarrollo local y la importancia del capital social (Buciega Arévalo, 2009).

El turismo tiene entidad suficiente para figurar entre las actividades económicas que gran prosperidad científica han proporcionado, unas sobre actividades turístico-deportivas (Luque Gil, 2003), otras centradas en el agroturismo (Cors Iglesias, 2003), turismo de retorno (Balcells Sespulgues, 2009) turismo activo (Rivera Mateos, 2010) y planificación estratégica en espacios turísticos interiores (Diez Santo, 2010).

También es interesante comprobar el interés mostrado por el paisaje en las contribuciones (Ibáñez González, 2001): la protección ambiental (Simancas Cruz, 2003), el paisaje forestal (Madrazo, 2007), el paisaje como patrimonio rural (Alberdi Collantes, 2001; Iranzo García, 
2009), la arquitectura vernácula (Gómez Reyes, 2007) así como las transformaciones espaciales (San Román Rodríguez, 2005) y articulación territorial (Menéndez Fernández, 2000; Heredia Laclaustra, 2011),

En relación con el turismo, pero también con la explotación del paisaje y otros recursos, es significativo el interés por la conservación del medio y las políticas de planificación aplicadas al medio rural tanto en zonas de especial protección de aves (Romero Calcerrada, 2001), red de caminos en zonas de montaña (Campillo Besses, 2001) como gestión de áreas de uso público en espacios naturales (Hidalgo Morán, 2006; Alberdi Nieves, 2011).

Persisten las tesis de corte agrarista centradas en la plurifuncionalidad de la agricultura (Moltó Mantero, 2005; Mollevi Bortoló, 2005), cooperativismo agrario (Basanta Fernández, 2002) así como aspectos más novedosos tales como la generación y reutilización de bioresiduos para uso agrícola (Pires Aguas, 2010). El agua y el sistema de regadío siguen siendo un tema en alza, necesario por ser un bien escaso, susceptible de uso múltiple y siempre alternativo (Torres Márquez, 2002; Ruiz Pulpón, 2005; Bouzaida, 2005, Férez Martinez, 2011). No faltan las tesis ganaderas (Borrás Ferrán, 2002; Barrachina Jiménez, 2011) y forestales (Martínez Garrido, 2000; Badia Perpinya, 2001; Moya García, 2004), así como la regulación de los sistemas agroalimentarios (Morales Pérez, 2002) y los sistemas productivos locales en la industrialización rural (Mecha López (2002).

Es obvio que se han producido cambios en la actividad agraria, pero también en la sociedad rural (Guirado González, 2001; Cortés Samper, 2006; Armas Quintá, 2009), de ahí el interés de analizar algunos de los fenómenos que presentan mayores dificultades tales como las tendencias demográficas y la despoblación (Estevez Adan, 2001; López Ruiz, 2007), la necesidad del dinamismo empresarial femenino (Nieto Figueras, 2003), la relación ruralurbana (Sempere Roig, 2005; Paül i Carril, 2006), la dinámica de la migración y sus implicaciones a través de los asentamientos neorrurales (Reinoso Moreno, 2009).

Un considerable grueso siguen siendo tesis con una visión ruralista enmarcadas en Iberoamérica. En la mayoría de los países latinoamericanos, la infraestructura universitaria local corresponde a las necesidades. De ahí, la carestía de buscar estos medios en universidades del extranjero, principalmente en lo que se refiere a la formación académica de los investigadores. Esta es la razón que lleva a profesores e investigadores a buscar los cursos de doctorado en universidades del Primer Mundo. De las 16 defendidas, México es el país con más aportaciones junto a Brasil. Le siguen Chile, Venezuela, Colombia y Costa Rica.

De manera aislada hay una tesis enmarcada en la enseñanza de geografía rural en Bachillerato (Miguel García, 2006).

Por término medio, según la base de TESEO, en España se defienden 6,5 tesis doctorales de «geografía rural» por año. En cuanto a la fecha de lectura, los años de mayor productividad son la primera mitad de la década, destacando 2002 y 2005 con más de 10 tesis. Esto puede influir porque en este período las normativas de los estudios universitarios de Tercer Ciclo han ido cambiando, pasando de los antiguos programas de doctorado, a la defensa de tesis y su consecuente obtención del Diploma de Estudios Avanzados (DEA), y a la situación actual de doctorados tras el master universitario. La adaptación al Espacio Europeo de Enseñanza Superior (EEES) se asienta sobre los fundamentos jurídicos establecidos por el RD 1393/2007, promulgado con el objetivo de ajustar los estudios españoles de postgrado a las nuevas directrices europeas. En el modelo anterior (reglamentado por el RD 778/1998) los 
estudios de doctorado incorporaban una parte de docencia y otra de investigación, mientras que el nuevo modelo se orienta hacia tareas de formación y desarrollo científico, que culminan en la tesis doctoral.

Si agrupamos las tesis en función de las Universidades en las que se leyeron, donde se han defendido más tesis rurales de Geografía es la Universidad de Barcelona, siendo la más productiva. En el siguiente bloque está la Universidad de Alcalá de Henares y Autónoma de Barcelona, y a cierta distancia la Universidad de Alicante y Santiago de Compostela.

Figura 2

PROCEDENCIA DE LAS UNIVERSIDADES DONDE SE LEEN LAS TESIS DOCTORALES «RURALES»

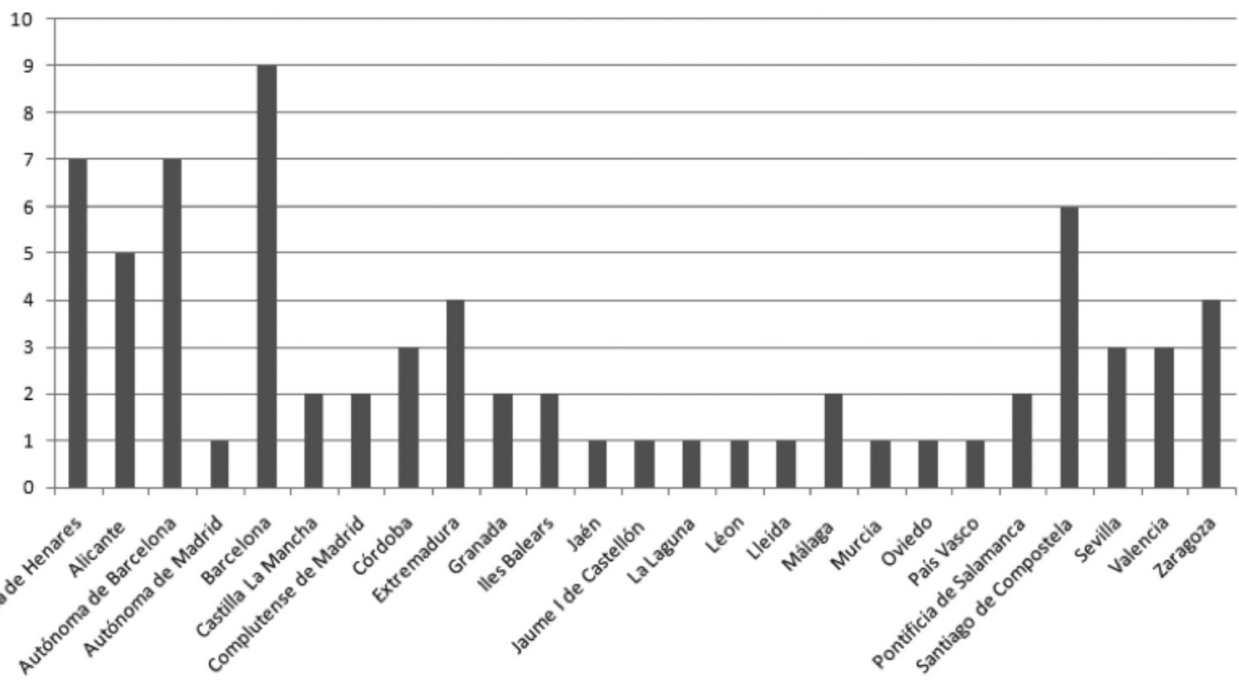

Fuente: Elaboración propia basada en Ministerio de Educación, Cultura y Deporte (2012).

Los directores de estas tesis doctorales son casi todos miembros del Grupo de Trabajo de la Asociación de Geógrafos Españoles (AGE) Rural, que han ido expandiendo sus líneas de investigación. Los que más han dirigido en esta primera década son Ruben Lois González, Antonio López Ontiveros, Francisco López Palomeque y Antoni Tulla Pujol. A éstos hay que añadir Joaquín Bosque Sendra, Javier Esparcia Pérez, Antonio Gil Olcina, Roser Mayoral Moliné, Javier Martínez Vega, Carmen Ocaña Ocaña, Patricio Rubio Romero y José Sancho Comins.

\section{LOS PROYECTOS DE INVESTIGACIÓN DE GEOGRAFÍA (2008-2011)}

La producción científica y tecnológica está determinada por los recursos económicos que el gobierno destina a las actividades de I+D y por la inversión en capital humano investigador. Uno de los factores que impulsa el progreso de la ciencia fue y es precisamente el intercambio de información. Por ello, la actividad investigadora llevada a cabo en los departamentos de Geografía españoles está sostenida en una parte sustancial por fondos consegui- 
dos de organismos públicos externos a las respectivas universidades mediante la concesión, previo concurso, de proyectos de investigación financiados (Canosa y otros, 2004)

Con el fin de analizar las investigaciones asociadas a los proyectos $\mathrm{I}+\mathrm{D}$ del antiguo Ministerio de Ciencia e Innovación y actual Ministerio de Economía y Competitividad del Gobierno de España, se seleccionaron todos los proyectos de Geografía de las últimas cuatro convocatorias (2008-2011): 101 proyectos concedidos (2008: 21, 2009: 20, 2010: 23, 2011 : 37). En algunos casos, entraña dificultad hacer distinción si son de geografía rural, ya que en ocasiones, lo rural se convierte en un adjetivo y no en término sustantivo. Esto sucede desde macro enfoques (espacio rural o reestructuración rural) a micro enfoques (espacio o economía rural de la comarca o región), y se aplica también con continuidad a enfoques o análisis sectoriales (turismo rural, industria rural, etc.) (Paniagua y Hoggart, 2002).

La evolución de los proyectos nacionales del área temática de Ciencias Sociales, en los que se encuentran insertos las Ciencias Políticas, Sociologia, Comunicación y Geografía, también ha sufrido cambios en estos cuatro años, ya que Comunicación se incorpora a éste en 2009. Sociologia es el campo donde más proyectos se conceden. El porcentaje de representación de la Geografía ha sido del 21,88\% (2008), 16,18\% (2009), 29,41\% (2010) y la proporción más alta, 31,62\% (2011).

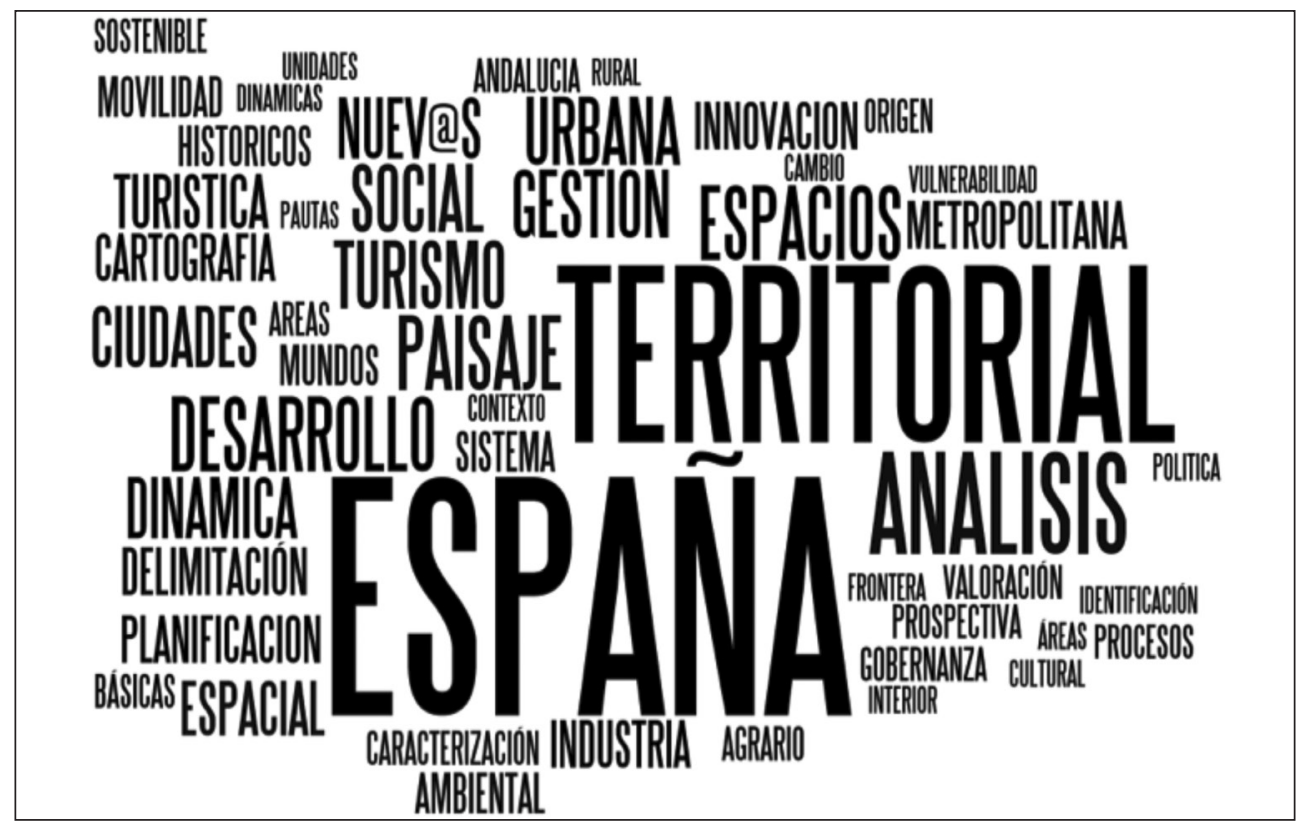

Fuente: Elaboración propia basada en la base de datos TESEO (2012).

El geógrafo debe ser un hombre de acción, un hombre de su tiempo, que se involucra con los problemas de la sociedad en que le ha tocado vivir (Olcina y Baños, 2004). El momento 
actual es importante, porque los problemas de carácter territorial, los que tienen que ver con las preocupaciones de la geografía, han adquirido una considerable presencia social en España y ello también se traduce en los proyectos de investigación. Los nuevos temas que se plantean en la disciplina (medio ambiente, paisaje, vulnerabilidad, innovación, movilidad o gobernanza) así lo requieren.

La Geografía está llamada a afrontar las cuestiones territoriales derivadas de la propia presencia del hombre sobre la Tierra, cuestiones que unas veces precisan un análisis detallado de los elementos del medio físico, otras, de los propios grupos sociales, y siempre un estudio de las formas de organización que elige el hombre en cada momento histórico en relación con su herencia cultural. En las investigaciones geográficas han cambiado los planteamientos metodológicos. Más allá de los análisis cuantitativos, correspondientes a un predominio de la epistemología positivista, se ha pasado a una influencia cada vez mayor de perspectivas culturales por la aplicación de métodos cualitativos (Woods, 2010).

La Universidad de Barcelona es el organismo que recibe más proyectos gracias a la investigación de sus miembros. Le sigue en el ranking la Universidad Autónoma de Barcelona. En tercera posición se sitúan la Universidad Autónoma de Madrid, Sevilla, CSIC, Complutense de Madrid y Alicante.

Figura 4

ORGANISMOS QUE RECIBEN PROYECTOS NACIONALES I+D DE GEOGRAFÍA EN ESPAÑA (2008-2011)

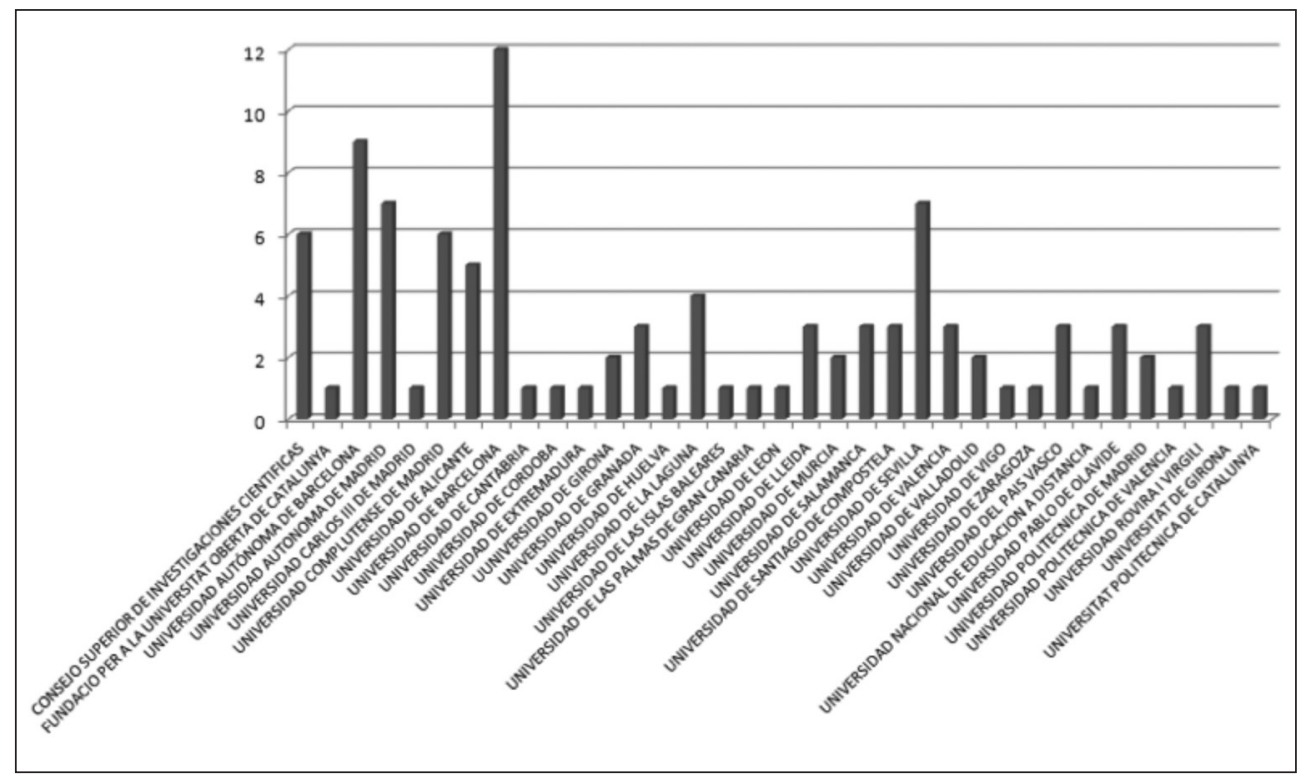

Fuente: Elaboración propia basada en Ministerio de Economía y Competitividad (2012).

Es cierto que en Ciencias Sociales la cuantia económica es escasa respecto a otras ciencias, y cada vez más se ha ido reduciendo su dotación. Los proyectos de 2008 recibieron un promedio de 75.000 euros, disminuyendo sus presupuestos, y alcanzando en el 2011 casi la 
mitad de los mismos (40.000 euros). También es representativa la orquilla presupuestaria máxima y mínima que hay entre los proyectos de dichos años. En el 2008 ésta se situaba entre 30.000 y 163.000 euros, acortándose estas cifras con el paso de los años, y alcanzando en 2011 de 11.000 a 88.000 euros. Esta disminución ha obligado a las universidades a solicitar más proyectos mantenidos por entidades regionales, provinciales o locales.

MEDIA DE LOS PRESUPUESTOS DE LOS PROYECTOS I+D CONCEDIDOS ENTRE 2008-2011

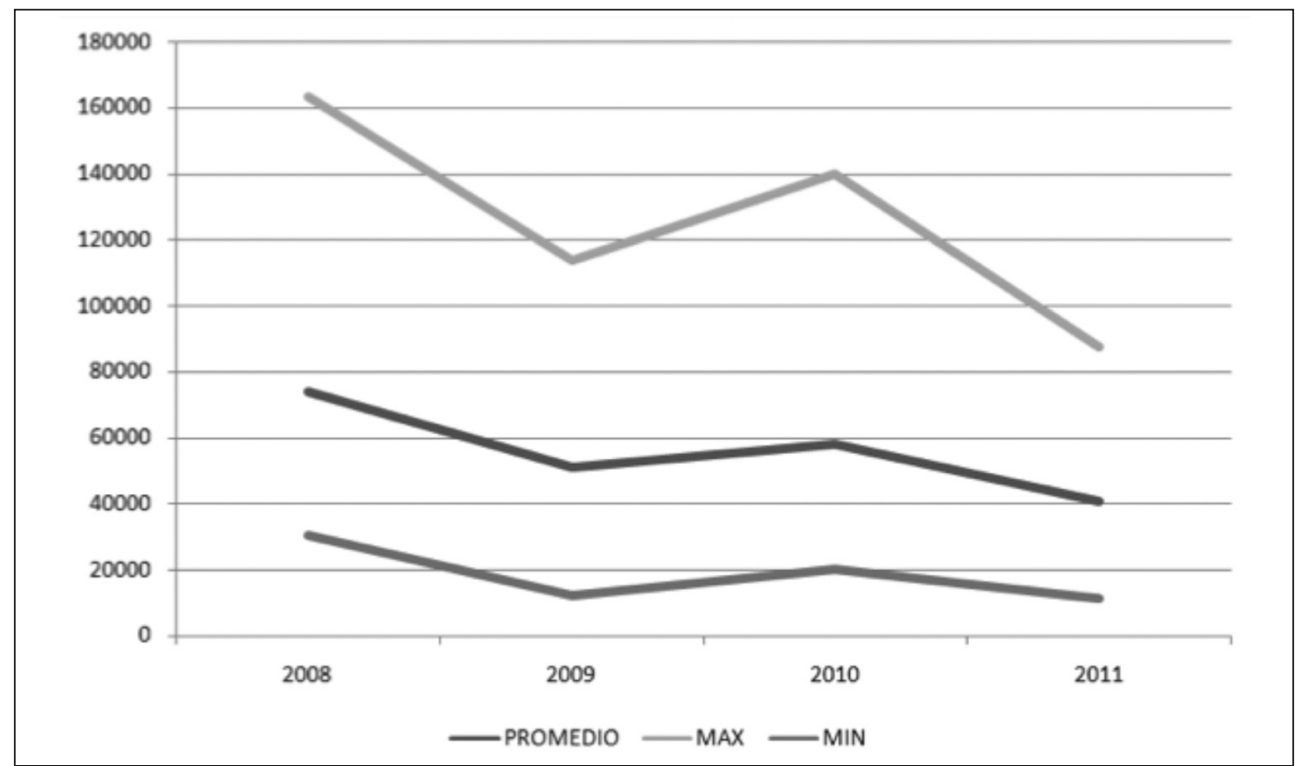

Fuente: Elaboración propia basada en Ministerio de Economía y Competitividad (2012).

\section{CONCLUSIONES}

En la primera década del siglo XXI, la geografía rural sigue estando abierta a otras disciplinas, y abarca nuevas tendencias metodológicas (la economía política, el giro cultural, el feminismo, el postmodernismo, etc.). Los estudios rurales han avanzado notablemente en su dispersión y complejidad. A la habitual difusión temática que encierra lo rural se suma la disparidad de perspectivas de estudios. Se plantean nuevos desafíos tales como la investigación de post-productivismo, el desarrollo rural sostenible, la prestación de servicios, y la percepción del paisaje (Clout, 2008).

Algunos de los avances científicos del desarrollo de la geografía rural son:

1) el interés que las investigaciones muestran por el cambio de la geografía agraria al desarrollo integrado de actividades primarias, industriales y terciarias de las zonas rurales,

2) los estudios de los geógrafos rurales han reducido su perspectiva clásica de la producción hacia un sistema espacial rural, que incluye el paisaje rural, la economía rural, 
la sociedad y las organizaciones públicas, a través de una reconsideración de la geografía del gobierno de áreas rurales. Todo ello ha sido dominado en buena medida por la cambiante naturaleza agraria, por la fragilidad de la producción y la generación de alternativas ligadas a los servicios verdes, pero también a nuevos modelos institucionales (Paniagua, 2007).

3) Estas investigaciones también han prestado atención a los vínculos y las interacciones entre zonas rurales y zonas urbanas (neorural, naturbanización, rururbano, etc.).

Y para seguir avanzado, cada vez más se investiga cómo afectan los grandes procesos de globalización a la supervivencia de lo rural, tanto desde una consideración espacial como social.

\section{BIBLIOGRAFÍA}

AGE (1991): Informe sobre resultados de la investigación en Geografía. [En línea], http:// age.ieg.csic.es/docs/01-00-result-inves.PDF. Consulta el 26 de octubre 2011.

AGE Rural (2000): Trayectoria histórica del grupo de Rural [En linea], http://age.ieg.csic.es/ geografía_rural/trayectoria_historica.html. Consulta el 25 de noviembre 2011.

CANOSA, E.; FROCHOSO, M.; MUÑOZ, J. (2004): «Los departamentos universitarios de Geografía y su producción cientifica». La Geografía española ante los retos de la sociedad actual. Comité español de la Unión Geográfica Internacional. Glasgow. 121-144.

CLOUT, H. (2008): «Thoughts on the evolution of rural geography in the British Isles «. Géocarrefour, Vol. 83/4, 2008, [En linea], http://geocarrefour.revues.org/7029. Consulta el 09 agosto 2012.

DE LÁZARO Y TORRES, M.L. (2002): «La Geografía a las puertas del tercer milenio a partir de las tesis doctorales leídas en los noventa». Anales de Geografía, 22: 49-66.

Ministerio de Economía y Competitividad (2012): Programa nacional de proyectos de investigación fundamental 2008-2011 [En linea], https://www.idi.mineco.gob.es. Consulta el 5 marzo 2012.

Ministerio de Educación, Cultura y Deporte (2012): Base de datos de Tesis Doctorales (TESEO). [En linea], https://www.educacion.gob.es/. Consulta el 25 enero 2012.

OLCINA, J.; BAÑOS, C.J. (2004): «Los fines de la geografía». Investigaciones Geográficas 33:39-62.

PANIAGUA, A. (2007): «Los flecos y lo relevante en los estudios rurales». Revista de Estudios sobre despoblación y desarrollo rural AGER 6: 183-192.

PANIAGUA, A.; HOGGART, K. (2002): «Lo rural, ¿hechos, discursos o representaciones? Una perspectiva geográfica de un debate clásico». Información comercial española, 803: $61-71$.

WOODS, M. (2010): «Performing rurality and practicing rural geography». Progress in Human Geography 34 (6): 835-846. 
